\title{
PATTERNED FABRIC DEFECT DETECTION USING A MOTIF-BASED APPROACH
}

\author{
Henry Y.T. Ngan, Student Member, IEEE, Grantham K.H. Pang, Senior Member, IEEE, and \\ Nelson H.C. Yung, Senior Member, IEEE
}

Department of Electrical and Electronic Engineering, The University of Hong Kong

\begin{abstract}
This paper proposed a patterned fabric defect detection method for sixteen out of seventeen wallpaper groups using a motif-based approach. From the symmetry properties of motifs, the energy of moving subtraction and its variance among motifs are mapped onto an energy-variance space. By learning the distribution of defect-free and defective patterns in this space, boundaries conditions can be determined for defect detection purpose. The proposed method is evaluated on four wallpaper categories, from which all 16 wallpaper groups can be generalized. Altogether, 160 defect-free lattices samples are used for learning the decision boundaries; and 200 other defect-free and 138 other defective samples are used for testing. An overall detection accuracy has reached 93.61\%, which outperforms previous approaches.
\end{abstract}

Index Terms - Wallpaper group, lattice, motif, patterned fabric, defect detection

\section{INTRODUCTION}

Patterned texture with periodic structures can be found in many daily items like wallpapers [1], ceramics [2], fabrics $[3,4]$, netting, ropes, chains, patterned metals, heated windows and other safety critical materials. It is composed of a fundamental unit called lattice [5], which exists by design. Usually, a patterned texture is synthesized by applying proper symmetry rules [5] of that lattice. In practice, when a lattice is replicated, various kinds of defects appear due to the manufacturing process. Traditionally, patterned texture after manufacturing is inspected by human, and rejected if the number of defects exceeds a threshold. Not only an automated defect detection system reduces the labor cost, it also ensures a high quality output in industrial automation and safety system. For example, any error of aircraft heated windscreen is fatal so that it is zero-tolerance on defects. This paper used patterned fabric as an example of defect detection for the industrial field. According to mathematicians, all the patterned textures can be classified into 17 wallpaper groups [5]. The patterned texture (Fig. 1(a)) in each wallpaper group is characterized by a prominent lattice (Fig. 1(b)).
Each lattice is further replicated by its basic component motif (Fig. 1(c)).

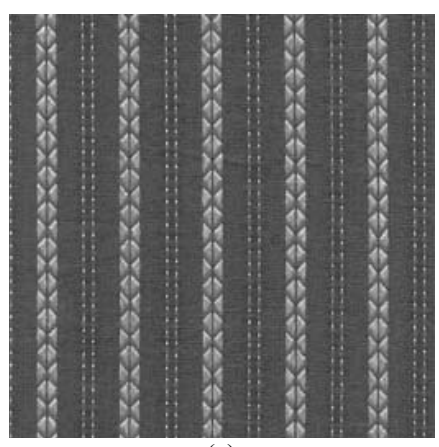

(a)

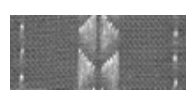

(b)

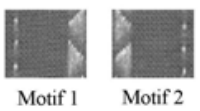

(c)

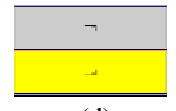

(d)
Fig. 1. (a) Real bookmark-patterned fabric of pm group;

(b) one possible lattice, (c) motifs of (b), (d) motif distribution of a lattice sample in [5]

However, most current fabric defect detection methods are pattern-oriented (non-motif-based), which means they do not rely on the presence of motif and are tested on one type of patterned texture only. The plain and twill fabrics [4] $[6,7]$ and dot-patterned fabric [8,9] are commonly analyzed. Moreover, none of the previous methods can claim to be applicable on other patterned textures. Furthermore, they can only classify if an input is defective or not, and fail to identify defects at a lattice or motif level. Hence, a generalized and localized defect detection approach is desirable.

To begin with, we first assume that the lattice extraction model constructed by Liu et. al [10] is used to identify a representative motif in the pattern. It is from this motif that our proposed method is based upon. In essence, energy of moving subtraction and its variance among motifs are calculated and mapped onto an energy-variance (EV) space, from which decisions are made as to whether a motif is defective or not. As all 16 groups of wallpaper can be easily transformed into three categories of motifs, by testing on these three categories, we can argue that all 16 groups can be handled.

The main contributions of the proposed method are:

(1) A generalized motif-based model is proposed for patterned fabric defect detection on 16 out of 17 wallpaper groups without requiring any modifications.

(2) It enables the decision boundaries to be determined through a set of defect-free samples. 
(3) At present, the defect detection can be accurate to the level of lattice. The overall detection success rate of the $\mathrm{pm}, \mathrm{p} 2$, pmm and $\mathrm{p} 4 \mathrm{~m}$ groups in this paper reach $93.61 \%$, which outperforms the previous methods.

This paper is organized as follows. Section II outlines the mathematical principle of proposed motif-based defect detection for patterned fabric. Section III depicts the detection results of $\mathrm{pm}, \mathrm{p} 2$, pmm and $\mathrm{p} 4 \mathrm{~m}$ wallpaper groups, respectively. At last, Section IV draws the conclusions for the proposed method.

\section{MATHEMATICAL PRINCIPLE}

The details of mathematical definitions and proofs are stated in [11]. For convenience, the key equations are outlined below.

\section{A. Lattice:}

A lattice is defined as $L=[f(a, b)]$ and a defective lattice, $\bar{L}$, is defined as $\bar{L}=L+P=[\bar{f}(a, b)]$ where $\bar{f}(a, b)=f(a, b)+\varepsilon(a, b)$ and $P=[\varepsilon(a, b)]$ is a $p \times q$ matrix that represents additive defects, and $\varepsilon(a, b) \in R, 0 \leq \bar{f}(a, b) \leq 1,1 \leq a \leq p$ and $1 \leq b \leq q$.

\section{B. Motif:}

A motif is defined as $M=[f(c, d)]$ and a defective motif, $\bar{M}$, is defined as $\bar{M}=M+P=[\bar{f}(c, d)]$ where $\bar{f}(c, d)=f(c, d)+e(c, d)$, and $P=[e(c, d)]$ is a $m \times n$ matrix that represents additive defects, and $e(c, d) \in R$, $0 \leq \bar{f}(c, d) \leq 1,1 \leq c \leq m$ and $1 \leq d \leq n$.

\section{C. k-norm Metric:}

Given any two motifs, $M^{s}$ and $M^{r}$ from lattice $L$, a k-norm metric is defined as $u^{s, r}=\left\|M^{s}-M^{r}\right\|_{k} / N$

$$
u^{s, r}=\left(\sum_{c=1}^{m} \sum_{d=1}^{n}\left|f^{s}(c, d)-f^{r}(c, d)\right|^{k}\right)^{\frac{1}{k}} / N,
$$

where $N=m \cdot n$. The $\mathrm{k}$ is set to be 1 in our application for simplicity. The total number of 1-norm metric for $u^{s, r}$ in one lattice is $C_{2}^{n}$, where $n$ is the number of motifs in a lattice. The $\mathrm{p} 1$ group is ineligible for k-norm metric definition since it has only one motif.

\section{Circular Shift Operation:}

Given any $m \times n$ motif

$$
M=[f(c, d)]=\left[\begin{array}{ccc}
f(1,1) & \cdots & f(1, n) \\
\vdots & & \vdots \\
f(m, 1) & \cdots & f(m, n)
\end{array}\right],
$$

a circular shift operation $H$ of a matrix produces $m \times n$ new motifs such that $M_{i j}=H[M]$ is defined as

$$
\begin{gathered}
M_{11}=\left[\begin{array}{ccc}
f(1,1) & \cdots & f(1, n) \\
\vdots & & \vdots \\
f(m, 1) & \cdots & f(m, n)
\end{array}\right], \cdots, M_{1 n}=\left[\begin{array}{ccc}
f(1, n) & \cdots & f(1, n-1) \\
\vdots & & \vdots \\
f(m, n) & \cdots & f(m, n-1)
\end{array}\right] \\
M_{m 1}=\left[\begin{array}{ccc}
f(m, 1) & \cdots & f(m, n) \\
\vdots & & \vdots \\
f(m-1,1) & \cdots & f(m-1, n)
\end{array}\right], \cdots, M_{m n}=\left[\begin{array}{ccc}
f(m, n) & \cdots & f(m, n-1) \\
\vdots & & \vdots \\
f(m-1, n) & \cdots & f(m-1, n-1)
\end{array}\right] .
\end{gathered}
$$

\section{E. Energy of Moving Subtraction:}

With motifs $M^{s}$ and $M^{r}$, the energy of moving subtraction is defined as

$$
K_{s, r}=\left(\sum_{i=1}^{m} \sum_{j=1}^{n} u_{i j}^{s, r}\right) / N=\sum_{i=1}^{m} \sum_{j=1}^{n}\left\|M^{s}-M_{i j}^{r}\right\| / N^{2},
$$

where $M_{i j}^{r}=H\left[M^{r}\right]$.

\section{F. Variance of Energy of Moving Subtraction:}

The variance of energy of moving subtraction, $K_{s, r}$, is defined as

$$
V_{s, r}=\sum_{i=1}^{m} \sum_{j=1}^{n}\left(K_{s, r}-u_{i j}^{s, r}\right)^{2} / N
$$

where $u_{i j}^{s, r}$ is 1-norm metrics of $M^{s}$ and $M^{r}$.

\section{G. Formulation of Decision Boundaries:}

Given the energy of moving subtraction and its variance, they form a $2 \mathrm{D}$ plane and we called it the $\mathrm{EV}$ space.

For $0 \leq \delta \leq\left|\bar{K}_{s, r}-K_{s, r}\right| \leq m \cdot n \cdot|e|$, and

$0 \leq \gamma \leq\left|\bar{V}_{s, r}-V_{s, r}\right| \leq \Phi$, a defect-free motif would fall into the following boundaries:

$$
\begin{aligned}
& K_{s, r}-\delta \leq K_{s, r} \leq K_{s, r}+\delta, \text { and } \\
& V_{s, r}-\gamma \leq V_{s, r} \leq V_{s, r}+\gamma,
\end{aligned}
$$

where $\delta \geq 0, \gamma \geq 0, \Phi=2\left(m n K_{s, r}+1\right)|e|+|e|^{2}$,

$$
|e|=\left|e^{s, r}\right|, e^{s, r} \in R[11] \text {. }
$$

If a $E V$ value of a lattice falls inside the boundaries, it is classified as defect-free (Fig. 2(a)); otherwise, it is considered as defect-free (Fig. 2(b)).

In summary, the proposed method contains two phases: phase of decision boundaries formulation on the EV-plane and phase of defect detection.

\section{DETECTION RESULTS OF SEVERAL WALLPAPER GROUPS}

\subsection{Detection Results of pm Group}

The bookmark-patterned fabric (Fig. 1) of the pm wallpaper group shown in this paper is used for evaluation here. Based on the lattice extraction model of Liu et. al [10], 100 defectfree and 25 defective lattice (Fig. 3) of size of $100 \times 42$ are 


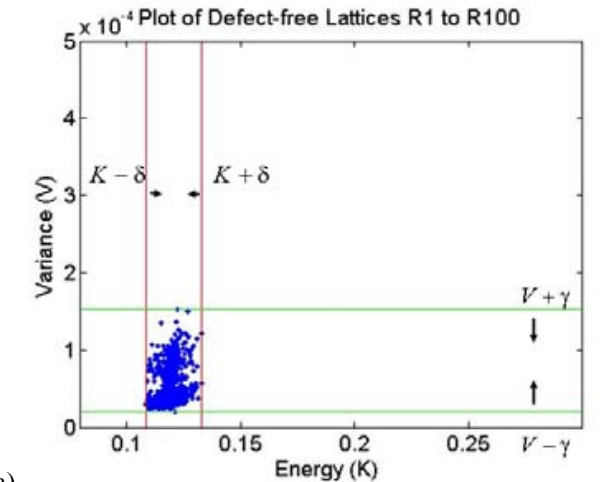

(a)

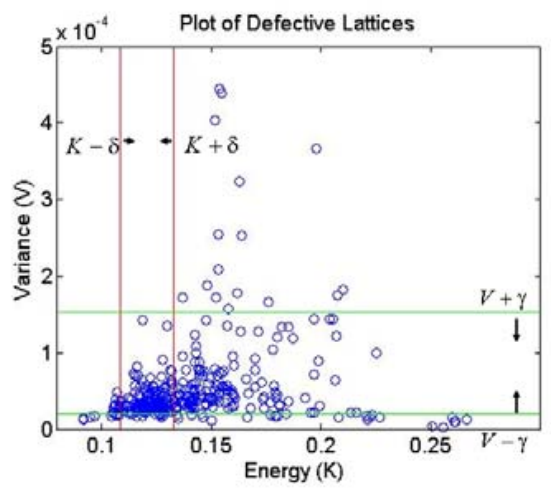

(b)

Fig. 2. (a) Boundaries and the EV plot of defect-free lattices;

(b) Boundaries and the EV plot of defective lattices.

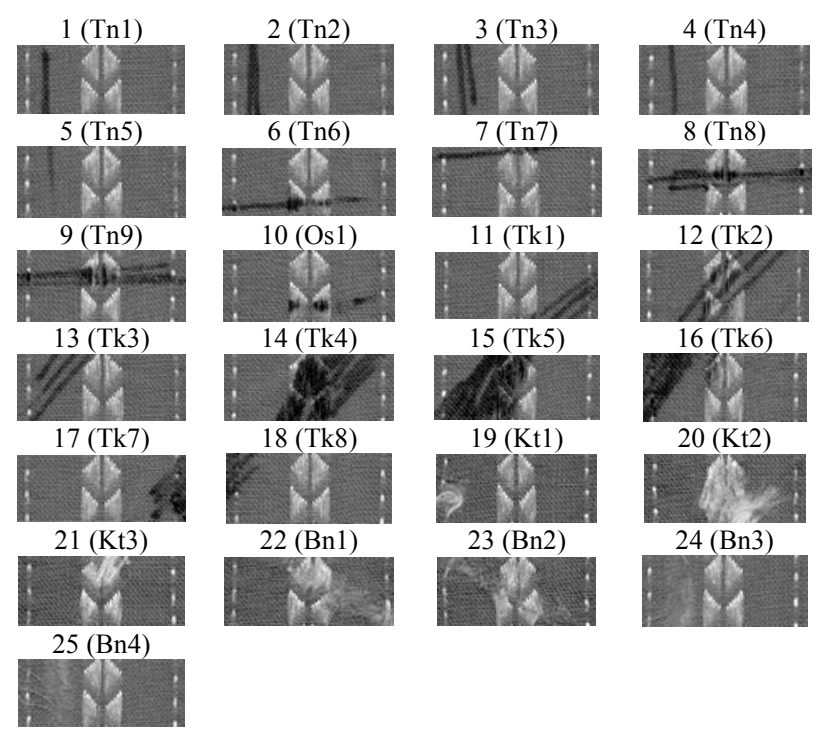

Fig. 3. 25 defective lattice samples, D1-D25, of size $100 \times 42$ of Bookmark-patterned fabric.

extracted manually from the fabric. The defective samples include the defects thin bar (Tn), oil stain (Os), thick bar $(\mathrm{Tk})$, knots $(\mathrm{Kt})$ and broken end $(\mathrm{Bn})$. The lattice sample in Fig. 1(d) shows the motif distribution of a pm group. The motif extraction for the bookmark-patterned lattices is based on the vertical axis of the lattice.

To formulate the decision boundaries, where there are four altogether, 40 lattices are randomly selected from 100 defect-free lattices, while the remaining 60 lattices and the 25 defective lattices are used for defect detection. There exists $C_{2}^{2}=1$ pair of energy of moving subtraction and its variance given as $\left\{K_{1,2}, V_{1,2}\right\}$ in the EV-plot. A total of 10 trials are performed for producing a general trend of the detection result of the pm group.

Two criteria are applied on this evaluation. First, if a point falls inside the boundaries of the EV-plane, then it is defect-free, else it is defective. Secondly, sensitivity (correct detection of defective lattices) is defined as Sensitivity $=T P /(T P+F N)$, and specificity (correct detection of defect-free lattices) is defined as Specificity $=T N /(T N+F P)$, where $T P=$ true positive, $T N=$ true negative, $F P=$ false positive and $F N=$ false negative. Furthermore, the detection success rate is defined as the percentage fraction of the number of correct detection of lattices over the total number of lattices, i.e. $(T P+T N) /(T P+F N+T N+F P)$.

Details of detection results are shown in Table I. The sensitivity is $93.20 \%$ and specificity is $95.17 \%$. The average overall detection success rate is $94.59 \%$. The best results are given in the $1^{\text {st }}, 2^{\text {nd }}, 8^{\text {th }}, 10^{\text {th }}$ trials $(96.47 \%)$ while the worst result is given in the $5^{\text {th }}$ trial $(90.59 \%)$. There are variations in detection results of the defect-free samples because the decision boundaries contract and relax depending on the randomly selected training samples.

TABLE I

TABle of AVERAGE DETECTION SuCCESS RATE. (USING 40 RANDOMLY SELECTED DEFECT-FREE TRAINING SAMPLES)

\begin{tabular}{cccc}
\hline $\begin{array}{c}\text { Set of } \\
\text { Data }\end{array}$ & $\begin{array}{c}\text { Sensitivity } \\
\text { TP/(TP+FN) } \\
\text { TP/25 }\end{array}$ & $\begin{array}{c}\text { Specificity } \\
\text { TN/(TN+FP) } \\
\text { TN/60 }\end{array}$ & $\begin{array}{c}\text { Detection Success } \\
\text { Rate }(\mathrm{n} / 85) \text { in } \%\end{array}$ \\
\hline \hline 1 & $23 / 25$ & $59 / 60$ & $82 / 85(96.47 \%)$ \\
\hline 2 & $23 / 25$ & $59 / 60$ & $82 / 85(96.47 \%)$ \\
\hline 3 & $23 / 25$ & $57 / 60$ & $80 / 85(94.12 \%)$ \\
\hline 4 & $24 / 25$ & $56 / 60$ & $80 / 85(94.12 \%)$ \\
\hline 5 & $23 / 25$ & $54 / 60$ & $77 / 85(90.59 \%)$ \\
\hline 6 & $24 / 25$ & $55 / 60$ & $79 / 85(92.94 \%)$ \\
\hline 7 & $24 / 25$ & $55 / 60$ & $79 / 85(92.94 \%)$ \\
\hline 8 & $23 / 25$ & $59 / 60$ & $82 / 85(96.47 \%)$ \\
\hline 9 & $23 / 25$ & $58 / 60$ & $81 / 85(95.29 \%)$ \\
\hline \hline 10 & $23 / 25$ & $59 / 60$ & $82 / 85(96.47 \%)$ \\
\hline \hline
\end{tabular}

\subsection{Detection Results of p2, pmm, p4m Groups}

Table II illustrates the motif shapes for 16 wallpaper groups, parallelogram, rectangle and triangle. All motif shapes can be transformed from irregular motifs to regular ones. Therefore, three wallpaper groups, p2, pmm and p4m groups (Fig. 4) are extracted as representative of each shape for further investigation.

Table III depicts the detection results of $\mathrm{p} 2$, pmm and $\mathrm{p} 4 \mathrm{~m}$ from their corresponding databases. Each of them use 40 defect-free lattice samples for formulating the decision boundaries. They are mutually exclusive from samples for defect detection. The detection success rates of $\mathrm{p} 2, \mathrm{pmm}$ 
TABLE II

MOTIF SHAPES FOR 17 WALLPAPER GROUPS

\begin{tabular}{|c|c|c|c|c|c|c|}
\hline Type & \multicolumn{2}{|c|}{ A } & \multirow{2}{*}{$\begin{array}{c}\text { B } \\
\text { Parallelogram }\end{array}$} & \multicolumn{3}{|c|}{$\mathrm{C}$} \\
\hline Motif Shape & Square & Rectangle & & Isosceles Triangle & $\begin{array}{c}\text { Half -isosceles } \\
\text { Triangle }\end{array}$ & $\begin{array}{c}\text { Equilateral } \\
\text { Triangle }\end{array}$ \\
\hline $\begin{array}{l}\text { Wallpaper } \\
\text { groups }\end{array}$ & $\mathrm{p} 4$, & $\begin{array}{c}\text { pm, pg, pmm, } \\
\text { pmg, cmm }\end{array}$ & $\mathrm{p} 1, \mathrm{p} 2, \mathrm{p} 3$ & $\begin{array}{c}\text { p4m, p4g, pgg, } \\
\mathrm{cm}, \mathrm{p} 31 \mathrm{~m}, \mathrm{p} 6\end{array}$ & p6m & $\mathrm{p} 3 \mathrm{~m} 1$ \\
\hline
\end{tabular}

(a)

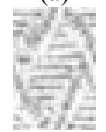

(d)

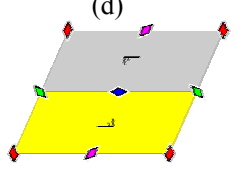

p2

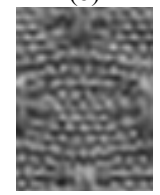

(e)

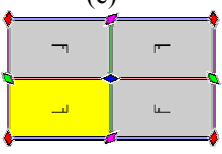

pmm

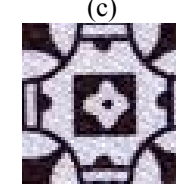

(f)

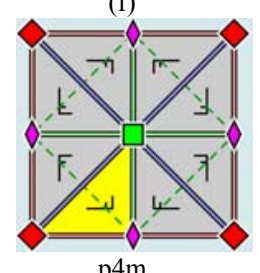

$\mathrm{p} 4 \mathrm{~m}$

Fig. 4. Real fabric lattice samples (a) Leaf-patterned in p2 group, (b) Dot-patterned in pmm group, (c) Coin-patterned in $\mathrm{p} 4 \mathrm{~m}$ group, (d), (e), (f) corresponding motif shapes [5] for each group.

TABLE III

TABLE of AVERAGE DETECTION SUCCESS RATE FOR PM, P2, PMM AND P4M GROUPS. RESULTS ARE AVERAGE FOR 10 TRIALS.

\begin{tabular}{cccc}
\hline \hline $\begin{array}{c}\text { Wallpaper } \\
\text { Group }\end{array}$ & $\begin{array}{c}\text { Sensitivity } \\
\text { TP/(TP+FN) }\end{array}$ & $\begin{array}{c}\text { Specificity } \\
\text { TN/(TN+FP) }\end{array}$ & $\begin{array}{c}\text { Detection Success } \\
\text { Rate in \% }\end{array}$ \\
\hline \hline \multirow{2}{*}{$\mathrm{pm}$} & $23.3 / 25$ & $57.1 / 60$ & $80.4 / 85$ \\
& $(93.20 \%)$ & $(95.17 \%)$ & $(94.59 \%)$ \\
\hline \multirow{2}{*}{$\mathrm{p} 2$} & $26 / 26$ & $37.3 / 40$ & $63.3 / 66$ \\
& $(100 \%)$ & $(93.25 \%)$ & $(95.91 \%)$ \\
\hline \multirow{2}{*}{$\mathrm{pmm}$} & $57.6 / 62$ & $55.9 / / 60$ & $113.5 / 122$ \\
& $(92.90 \%)$ & $(93.12 \%)$ & $(93.03 \%)$ \\
\hline \multirow{2}{*}{$\mathrm{p} 4 \mathrm{~m}$} & $21.6 / 25$ & $37.6 / 40$ & $59.2 / 65$ \\
& $(84 \%)$ & $(94 \%)$ & $(91.08 \%)$ \\
\hline \hline \multirow{2}{*}{ Average } & $128.5 / 138$ & $187.9 / 200$ & $316.4 / 338$ \\
& $(93.12 \%)$ & $(93.95 \%)$ & $(93.61 \%)$ \\
\hline \hline
\end{tabular}

and $\mathrm{p} 4 \mathrm{~m}$ groups are 95.91\%, 93.03\%, and $91.08 \%$, respectively.

\section{CONCLUSIONS}

A generalized motif-based method for patterned fabric defect detection has been presented in this paper, and the pm, p2, pmm, p4m wallpaper groups have been evaluated. The overall defect detection success rate for these 4 groups is $93.61 \%$, which outperforms all previously published approaches. Since p2, pmm, p4m groups are representatives of 3 motif shapes in 16 wallpaper groups, their successful results implies that all remaining wallpaper groups can be handled similarly.

\section{ACKNOWLEDGMENTS}

Dr. G. Pang would like to thank URC/CRCG small project funding account 10205131 for financial support to this research work.

\section{REFERENCES}

[1] K. Castro, P. Vandenabeele, M.D. Rodriguez-Laso, L. Moens, J.M. Madariaga, "Improvements in the wallpaper industry during the second half of the $19^{\text {th }}$ century: MicroRaman spectroscopy analysis of pigmented wallpapers," Spectrochimica Acta, Part A, vol. 61, pp. 2357-2363, 2005.

[2] M.L. Smith and R.J. Stamp, "Automated inspection of textured ceramic tiles," Computer In Industry, vol. 43, pp. 73-82, 2000.

[3] L. Tao, P. Witty and T. King, "Machine Vision in the Inspection on Patterned Textile Webs," Industrial Inspection ( Digest No:1997/041 ), IEE Colloquium, pp.9/1-9/5, 10 Feb 1997.

[4] H. Sari-Sarraf and J.S. Goddard, "Vision System for Onloom Fabric Inspection," IEEE Trans. Ind. Applicat., vol. 35, no. 6, pp. 1252-1259, 1999.

[5]Wikipedia, http://en.wikipedia.org/wiki/Wallpaper_group, 2007.

[6] M. Bennamoun and A. Bodnavova, "Automatic visual inspection and flaw detection in textile materials: Past, Present, and Future," Proc. IEEE SMC, pp. 4340-4343, 1998.

[7] C. H. Chan and G.K.H. Pang., "Fabric Defect Detection by Fourier Analysis," IEEE Trans. Ind. Applicat., vol.36, no.5, Sept/Oct 2000.

[8] H.Y.T. Ngan and G.K.H. Pang, "Novel Method for Patterned Fabric Inspection using Bollinger Bands," Opt. Eng., vol. 45, 087202, pp.1-15, 2006.

[9] H.Y.T. Ngan, G.K.H. Pang, S.P. Yung and M.K. Ng, "Wavelet Based Methods on .Patterned Fabric Defect Detection," Pat. Rec., vol. 38, no. 4, pp. 559-576, 2005.

[10] Y. Liu, R.T. Collins and Y. Tsin, "A Computational Model for Periodic Pattern Perception Based on Frieze and Wallpaper Groups," IEEE Trans. PAMI, vol. 26, no. 3, pp. 354-371, 2004.

[11] H.Y.T. Ngan, G.K.H. Pang and N.H.C. Yung, "Motifbased Defect Detection on Patterned Fabric," submitted 2007. 